\title{
Monitoring the Behavioral and Adrenal Activity of Giraffe (Giraffa camelopardalis) to Assess Welfare During Seasonal Housing Changes
}

\author{
Catherine B. Razal ${ }^{1}$, Jocelyn Bryant ${ }^{1}$, and Lance J. Miller ${ }^{1}$
}

${ }^{1}$ Chicago Zoological Society, Brookfield Zoo, Brookfield, IL

* Corresponding author (Email: Catherine.Razal@czs.org)

Citation - Razal, C. B., Bryant, J., \& Miller, L. J. (2017). Monitoring the behavioral and adrenal activity of giraffe (Giraffa camelopardalis) to assess welfare during seasonal housing changes. Animal Behavior and Cognition, 4(2), 154-164. https://doi.org/10.12966/abc.03.05.2017

\begin{abstract}
In inclement weather, northern zoos are required to provide animals from warmer climates with indoor exhibits. These indoor exhibits are typically smaller, lack natural substrate, and have lower levels of stimulation for the animals. The purpose of this study was to examine the welfare of four reticulated giraffe (Giraffa camelopardalis reticulata) exhibited at the Chicago Zoological Society - Brookfield Zoo - during the summer in an outdoor enclosure compared to the winter in an indoor enclosure. A combination of direct behavioral observati ons, percentage of time spent recumbent, and adrenal hormone monitoring through fecal samples were utilized for a more comprehensive look at welfare. Individual variation was observed between the giraffe. An adult female giraffe engaged in Active Forage behavior significantly more in the winter compared to summer (mean difference $=0.23, p$ $=0.049)$. An adult female and a juvenile male displayed significantly more Active Non-Forage behavior in the summer than in the winter ( mean difference $=0.32, p=0.048$; mean difference $=0.31, p=0.048$ ). The predominant behavior in the summer for the group as a whole was Active Non-Forage (59\%), whereas Active Forage was most prevalent in the winter $(60 \%)$. There was also a significant positive correlation between time spent recumbent per hour for each 24-hour day measured in the summer and winter $(r=0.51, p=0.010)$. Although no significant differences were found for individual FGM concentrations between the seasons, average FGM concentrations for the group were $2306.67 \mathrm{ng} / \mathrm{g}$ in the summer and $4261.64 \mathrm{ng} / \mathrm{g}$ in the winter. Information gained from this study can help aid animal managers to make informed decisions to ensure each individual giraffe is thriving year-round. In addition, we hope this study can serve as a model to examine the seasonal welfare of other animals in zoological institutions with similar conditions.
\end{abstract}

Keywords - Giraffe, Enclosure, Animal behavior, Fecal glucocorticoid metabolites, Recumbency, Animal welfare

There are many factors related to zoo enclosures that may impact an animal's overall welfare. Whether it involves enclosure space and design (Mallapur, Qureshi, \& Chellam, 2002; Ross, Calcutt, Schapiro, \& Hau, 2011) or increasing environmental enrichment into an animal's exhibit (Mellen \& Sevenich MacPhee, 2001), exhibit characteristics and complexity are at the forefront in recent research in zoos. More studies are now examining how animals utilize their enclosures based on a 24-hour period, because many species spend time in different exhibits depending on time of day (Posta, Huber, \& Moore, 
2013). However, there is little information available on the welfare of animals that utilize different exhibits depending on season.

Northern climate zoos are required to provide indoor exhibits for species native to warmer temperatures to protect them from inclement weather. These indoor exhibits are typically smaller, lack natural substrate, and offer lower levels of stimulation when compared to exhibits utilized in the summer months (Novak, O’Neill, \& Suomi, 1992; Ross et al., 2011; Ross, Schapiro, Hau, \& Lukas, 2009). Concerns surrounding diet, activity, and overall welfare have been raised about keeping animals in these different conditions (Hoff, Powell, Lukas, \& Maple, 1997; Posta et al., 2013). Previous research suggests that housing animals indoors may have a significant impact on activity levels and overall behavior. Hoff et al. (1997) demonstrated that lowland gorillas (Gorilla gorilla gorilla) display more social behaviors when inside compared to their outdoor exhibit. Macedonia (1987) found that sifakas (Propithecus verreauxi) that were housed outdoors were more active than those housed indoors. Similarly, one study involving African elephants (Loxodonta africana) found that the elephants were more active in their outdoor exhibit when compared to their inside enclosure (Posta et al., 2013). The results of these studies demonstrate how varying enclosures can potentially influence an animal's behavior.

Current trends in animal welfare research in zoos highlight the importance of using multiple welfare indicators in order to better understand how animals adapt to varying environments (Walker, Diez-Leon, \& Mason, 2014; Whitham \& Wielebnowski, 2013). Several studies have demonstrated that using hormone monitoring in conjunction with behavioral measures provide a better overall picture and understanding of welfare (Shepherdson, Carlstead, \& Wielebnowski, 2004; Shepherdson, Lewis, Carlstead, Bauman, \& Perrin, 2013; Wielebnowski \& Brown, 2003). Non-invasive hormone monitoring, such as measuring glucocorticoids (GC), can provide valuable insight into how an animal responds and adapts with its environment (Baird et al., 2016; Schwarzenberger, 2007; Wielebnowski, 2003). GC are one of the major components of the stress response (Sapolsky, 1992) and GC monitoring has been documented in previous research as a useful tool for managing animals in professional care. Fanson and Wielebnowski (2013) found that size of the enclosure was one of the main factors that correlated with GC secretion in Canadian lynx. In this study, one individual had lower GC concentrations when moved from a small enclosure to a significantly larger one. Effects of season on GC concentrations has been observed in zoo giant pandas, in which an individual in one study had higher GC concentrations in the winter and spring than in the summer season (Owen, Czekala, Swaisgood, Steinman, \& Lindburg, 2005). Both studies used GC collection techniques that were non-invasive, one through fecals (Fanson \& Wielebnowski, 2013) and the other through urine (Owen et al., 2005). These methods are valuable techniques and potentially more accurate by not having increased GC secretion caused by captureinduced stress (Millspaugh \& Washburn, 2004; Millspaugh et al., 2001).

In addition, another valuable measure of welfare that relates to varying enclosures is the amount of opportunity the animal has to rest. There are several studies involving dairy cattle that demonstrate the welfare implications of rest and recumbency. For example, inadequate resting time (i.e., recumbency) has been linked to states of chronic stress in cattle (Ladewig \& Smidt, 1989). In one study, cattle had higher rumination levels when lying down (Hassall, Ward, \& Murray, 1993), which is an important factor as rumination is thought to occur when animals are in a calm state (Orban, Siegford, \& Snider, 2016; Trunkfield \& Broom, 1990). Haley, Rushen, and de Passillé (2000) examined cattle under two different types of housing systems, and found that the subjects spent more time recumbent in the large pens when compared to the smaller ones. However, with all of the extensive research involving recumbency and cattle, there are few studies involving other species with the exception of horses (Tucker, Weary, \& Fraser, 2004) and more recently, elephants (Holdgate et al., 2016). Literature involving recumbency involving similar species, such as giraffe (Giraffa camelopardalis), is limited and mostly reported in wild populations (Pellew, 1984). Although there is limited research outside of the agricultural field, these studies demonstrate how recumbency can be an important indicator of welfare for other species.

Studies examining the impact of exhibit size and differences of indoor and outdoor enclosures are becoming more prominent in zoological institutions (Lukas, Hoff, \& Maple, 2003; Posta et al., 2013; Ross \& Shender, 2016). However, there is still a lack of literature on exhibiting animals in different 
enclosures due to seasonal weather. Giraffe are one of the most common species in zoo collections. Many northern zoos house giraffe in two separate spaces depending on season, although little information is available regarding whether they experience behavioral variation between these two seasonal exhibits. Similarly to cattle, giraffe behavior consists of high foraging levels (Duggan, Burn, \& Clauss, 2015; Hatt et al., 2005). Wild populations of giraffe are reported to spend about $60 \%$ of the daytime foraging (Bashaw, Tarou, Maki, \& Maple, 2001; Pellew, 1984). Today, many zoos provide browse to promote natural foraging behaviors for giraffe, and use a combination of alfalfa hay and concentrated feeds (e.g., grain, pellets) to maintain adequate nutrition (Duggan et al., 2015; Myers, 2004). During winter months, many northern zoos with colder climates have a reduced availability of browse. During this time, a giraffe's diet would consist of mostly items (e.g., pellets, hay) that do not promote natural tongue manipulation and could lead to increases in oral stereotypies (Duggan et al., 2015; Hummel, Clauss, Baxter, Flach, \& Johansen, 2006; Schaub et al., 2004). Bashaw et al. (2001) found that oral stereotypies (i.e., repetitive licking of non-food objects) were the most common stereotypic behavior of giraffe in North American zoos (Bergeron, Badnell-Waters, Lambton, \& Mason, 2006). The authors suggested that environmental variables, such as limited exhibit space, may have a strong influence on abnormal behavior (Bashaw et al., 2001). These results further highlight the importance of examining whether housing giraffe in smaller enclosures during the winter will impact behavior and welfare.

The purpose of this study was to examine the welfare of giraffe during the summer in an outdoor enclosure compared to the winter in an indoor enclosure. Behavioral observations, recumbency monitoring with accelerometer data loggers, and fecal glucocorticoid monitoring (FGM) through fecal samples allow for a more comprehensive look at the welfare of giraffe. Based on similar studies involving cattle (Haley et al., 2000; Ladewig \& Smidt, 1989), we specifically wanted to examine if giraffe in the outdoor enclosure will have greater behavioral diversity and higher recumbency rates, and if there would be significant differences in FGM concentrations across seasons. Information gained from this research can help inform animal care and management to help ensure each individual is thriving year-round and can serve as a model for other species housed in similar conditions.

\section{Method}

\section{Study Animals}

The subjects of this study included four reticulated giraffe (Giraffa camelopardalis reticulata) exhibited at the Chicago Zoological Society - Brookfield Zoo, Brookfield, IL. The subjects included a 10year old dam (G2), her 2-year old male calf (G4), and two unrelated females, one at 8 years old (G1) and one at 25 years old $(\mathrm{G} 3)\left(M_{\text {age }}=11.3\right.$ years $) . \mathrm{G} 1$ and G4 were born at the study institution, whereas G2 and G3 were born at other zoological institutions and moved to the study institution at two years and one year of age, respectively.

\section{Housing and Management}

The giraffe habitat includes two exhibits, a summer outdoor exhibit and an indoor winter exhibit. The giraffe are given access to their outdoor exhibit 24 hours a day in the summer months. The outdoor exhibit measures $4130 \mathrm{~m}^{2}$, and includes dirt and grass substrate with natural vegetation surrounding the exhibit. The outdoor exhibit has multiple public viewing areas, as well as a pool on the east side of the exhibit totaling $251 \mathrm{~m}^{2}$ with a maximum depth of two meters. During the summer months, leafy browse (e.g., varieties of mulberry, willow, honey locust, silver maple, sugar maple) and grain were offered twice a day along with piles of alfalfa hay strung up in baskets around the exhibit. In addition, the giraffe are provided outdoor enrichment in the form of food enrichment devices.

During the winter months, the giraffe have access only to the indoor enclosure and indoor holding stalls. The indoor enclosure is adjacent to the outdoor enclosure, measuring $153 \mathrm{~m}^{2}$. The floor in the indoor enclosure is concrete supplemented with a combination of rubber mats and wood chips. Behind the 
indoor enclosure are three indoor holding stalls, all measuring approximately $34 \mathrm{~m}^{2}$, and one indoor stall with a giraffe restraining device, measuring $18 \mathrm{~m}^{2}$. During the winter, the giraffe were given non-leafy browse branches (e.g., varieties of mulberry, willow, honey locust, silver maple, sugar maple) and grain twice a day, along with alfalfa hay available all day. Different types of enrichment were offered in the indoor enclosure, including various types of tongue-manipulating objects (e.g., spoons, metal fixtures).

Every morning, the giraffe are brought inside to the indoor holding stalls for either indoor or outdoor exhibit servicing, depending on the season. While in their indoor stalls, the giraffe receive their morning feed and participate in training sessions. Training sessions are held to prepare the animals for voluntary participation in husbandry and veterinary procedures.

\section{Behavioral Data Collection}

Behavioral observations were conducted at two different times in a year. August 3, 2015 to August 28, 2015 represented the summer data collection, and January 25, 2016 to February 19, 2016 represented the winter data collection. Focal observations lasted 15 minutes and behavioral data were collected using a combination of all occurrence sampling and instantaneous sampling at one minute intervals (Altmann, 1974). Observations took place five days a week between 10:00 $\mathrm{h}$ and 16:00 $\mathrm{h}$ using a randomized schedule calculated from Microsoft Excel (Microsoft Corporation, Redmond, WA). Each of the four subjects were observed a total of four times a day: twice in the morning and twice in the afternoon resulting in 80 observations per subject per season, and a total of $2400 \mathrm{~min}$ ( $40 \mathrm{hrs}$ ) of data for each subject during the entire study.

\section{Recumbency Monitoring}

The percentage of time in which subjects were recumbent was examined using a HOBO Pendant $\mathrm{G}$ data logger (Onset Computer Corporation, Pocasset, MA). The data logger is a three-axis accelerometer with an eight-bit resolution and records $\mathrm{x}-, \mathrm{y}-$, and $\mathrm{z}$-axis acceleration readings. Although this was the first time data loggers were used on giraffe, these loggers have been used extensively with cattle to measure recumbency as an indicator of animal welfare (Medrano-Galarza, Gibbons, Wagner, de Passillé, \& Rushen, 2012). The data loggers are approximately $60 \mathrm{~mm}$ in length, $33 \mathrm{~mm}$ in width, and $23 \mathrm{~mm}$ deep, and were placed in a pouch hand-stitched to an elastic band that was attached to the subject's ankle by Velcro. Because the subjects participate in tactile training every morning, training for desensitization to the bands occurred prior to the summer study period and took only one month to complete. The bands with the data loggers were placed on each subject once a week during August and February for a 24-hour period, resulting in a total of eight 24 -hour periods (192 hrs) per individual during the study. The data loggers were programmed using HOBOware $@$ Software Version 3.7.4 (Onset Computer Corporation, Pocasset, MA) to launch and record using a logging interval of one minute.

\section{Fecal Hormone Extraction and Assay Analyses}

Fecal samples were collected by animal care staff approximately three times a week during each study month. Subjects were observed by animal care staff and volunteers during the day to record defecation locations. The fecal sample was collected within an hour of defecation and then stored in a freezer at $-20^{\circ} \mathrm{C}$ until the time of analysis. There were a total of 86 samples (range $20-23$ per individual) collected during the study.

Fecal glucocorticoid metabolites (FGM) were extracted using $80 \%$ ethanol in $\mathrm{dH} 2 \mathrm{O}$. First, $0.5 \mathrm{~g}$ $( \pm 0.05 \mathrm{~g})$ of each wet fecal sample was weighed out into $16 \times 125 \mathrm{~mm}$ polypropylene tubes (Mettler balance, model \#AB104-5). Then, $5 \mathrm{ml}$ of $80 \%$ ethanol solution was added to each extraction tube. Each tube was vortexed and placed on a rotator (Fisher Labline Maxi Rotator, model \#4631) overnight (14 - 18

hrs). Tubes were then centrifuged for $15 \mathrm{~min}$ at $1500 \mathrm{rpm}$ (Marathon 3000R centrifuge, model \#120). For each sample, $1 \mathrm{ml}$ of supernatant was pipetted into $1 \mathrm{ml}$ of assay buffer $(0.1 \mathrm{M}$ phosphate buffered saline 
containing $1 \% \mathrm{BSA}, \mathrm{pH} 7.0$ ) into $12 \times 75 \mathrm{~mm}$ polypropylene tubes to produce a 1:10 dilution. Extracts were stored frozen at $-20^{\circ} \mathrm{C}$ until assay analyses.

The validation techniques used in this study for FGM analysis have been previously used in other studies (Bashaw et al., 2016; Chinnadurai et al., 2009; Morrow, Kolver, Verkerk, \& Matthews, 2002; Yadav et al., 2013), but obtaining a biological validation (i.e., an ACTH challenge) for this study was not possible. We validated the assay biochemically for this species and monitored variability between assays using quality high and low controls. Biochemical validation of the assays consisted of the following: 1 . parallelism with the standard curve, and 2. recovery to determine the percentage of exogenous hormone measured. To establish parallelism, serial two-fold dilutions of a sample pool (1:10 - 1:5120) were tested for potential interference in the sample matrix, linearity with the standard curve, and to determine the appropriate dilution factor at which to run the samples. The optimal dilution factor was 1:200 as this dilution was the closest to $50 \%$ binding of the sample pool (Figure 1). Recovery of exogenous hormone was measured by spiking one baseline diluted sample with each of the 5 highest standards in separate tubes, each standard containing a known amount of hormone $(250-4000 \mathrm{pg} / \mathrm{mL})$. The average percent recovery was calculated by dividing the measured concentration of hormone by the expected concentration of hormone multiplied by 100 .

Enzo Life Sciences supplied the corticosterone commercial EIA kit used to analyze FGM concentrations (Ann Arbor, MI, USA, catalog \# 901-097). The manufacturer supplied all needed instructions and components. We read plates on a photospectrometer plate reader (Dynex MRX Revelation) at $405 \mathrm{~nm}$. The cross-reactivity of the Enzo Life Sciences corticosterone anti-body are 100\% corticosterone, $28.6 \%$ desoxycorticosterone, $1.7 \%$ progesterone, $0.28 \%$ tetrahydrocorticosterone, $0.18 \%$ aldosterone, $0.13 \%$ testosterone and any other steroids were $<0.05 \%$. Assay sensitivity was $26.99 \mathrm{pg} / \mathrm{mL}$ and the intra-assay coefficient of variation was $3.90 \%$ at $44.00 \%$ binding with an average concentration of $521.70 \mathrm{pg} / \mathrm{mL}(n=8)$. Inter-assay variation was determined using a high and low control, $6.67 \%$ at $27.58 \%$ binding and $6.84 \%$ at $51.66 \%$ binding, respectively. The average recovery of exogenous corticosterone was $106.93 \%(S D=12.49 \%)$. We expressed all hormone data in $\mathrm{ng} / \mathrm{g}$ wet feces.

\section{Statistical Analysis}

Behavioral data. Behavioral states and behavioral diversity were examined for both individuals and as a group between seasons. Behavioral states coded from instantaneous sampling were used to determine behavioral diversity. Behavioral diversity can be used as a positive indicator of welfare as research has shown that individuals that have a varied repertoire of natural behaviors have a greater adaptability to environmental change (Swaisgood, 2007). Previous research has also demonstrated an inverse relationship between behavioral diversity and FGM concentrations (Miller, Pisacane, \& Vicino, 2016). Behavioral diversity was calculated using the Shannon-Wiener diversity index because of its ability to evaluate both the richness (number of behavioral states) and the evenness (distribution of behavioral states) in a dataset (Hacker, 2015; Shannon \& Weaver, 1949).

Based on non-normality and a small-sample size $(N=4)$, phase design randomization tests were used (Dugard, File, \& Todman, 2012). Randomization tests are appropriate and often utilized during a single subject design (Kratochwill, 2013), and consider measurements on a series of occasions in each phase (Dugard et al., 2012). In our study, the daily behavioral states were measured with the phases being summer and winter. Behavioral states were combined and simplified for the randomization tests to reduce the chance of Type 1 error: Active Forage (Feed, Ruminate), Active Non-Forage (Locomote, Bend, Stand Alert, Stand Non-Alert, Play, Social Positive, Explore/Interact Enrichment, Explore/Interact Environment, Self-Maintenance, Keeper Interaction, Visitor Interaction), Inactive (Rest/Sleep), and Abnormal (Abnormal) (Table 1). Inactive (Rest/Sleep) was a behavioral state measured separately from the recumbency data. In addition, Social Negative and Pace behaviors were not observed during the study and were removed from further analysis. Mean differences were calculated from the randomization tests. All behavioral data were corrected for proportion of time visible, and data from each day were averaged to create one data point per individual animal. In addition, average percentage of time visible of combined 
behavioral states were calculated for each individual (Figure 2), as well as group averages. Furthermore, all occurrence behavioral data were used to calculate rates of Play and Abnormal behavior per individual and for the group from summer to winter (number of behavioral events/time visible).

Recumbency data. Data were exported from the loggers using HOBOware $\odot$. Data were entered into a Microsoft Excel template and were examined using a macro previously validated in cattle (Gibbons, Medrano-Galarza, de Passillé, \& Rushen, 2012; Medrano-Galarza et al., 2012) and elephants (Holdgate et al., 2016). Percentage of lying bouts (recumbency) for each giraffe was calculated during hours of day $(600 \mathrm{~h}$ to $1800 \mathrm{~h})$ and night $(1800 \mathrm{~h}$ to $600 \mathrm{~h}$ ). Phase design randomization tests were also used to examine individuals and as a group for significant differences in recumbency across seasons. Hourly recumbency was calculated for each individual hour for each subject. A correlation coefficient was used to explore the relationship between time spent recumbent per hour in each 24-hour day measured $(n=8)$ in the summer and winter.

FGM analysis data. Average FGM concentrations (ng/g) were calculated for each individual and as a group for both seasons. A phase design randomization test was used to determine any differences in individual FGM concentrations from summer to winter.

For all statistical tests, alpha levels were considered significant at $p<0.050$.

\section{Results}

\section{Behavioral Data}

Active Non-Forage was the most predominant behavior in the summer study period (group average $=59 \%$ of visible time observed), whereas Active Forage was most prevalent in the winter study period (group average $=60 \%$ of visible time observed) (Figure 2). However, there were no significant differences in behavior for the group when comparing summer to winter (Table 2). Mean differences for the group and individuals from the phase design randomization tests are presented in Table 2. One subject, G2, engaged in the behavior Active Forage significantly more in the winter when compared to summer (mean difference $=0.23, p=0.049$ ). Average proportion of time spent for the individual behavioral states in the combined state Active Forage were calculated for G2: Feed (summer $=39 \%$; winter $=64 \%$ ) and Ruminate (summer $=13 \%$; winter $=10 \%$ ). Two subjects, G3 and G4, displayed significantly more Active Non-Forage behavior in the summer than in the winter (for G3, mean difference $=0.32, p=0.048$; for $\mathrm{G} 4$, mean difference $=0.31 ; p=0.048$ ). Average proportion of time spent for the individual behavioral states in the combined state Active Non-Forage were calculated for G3 and G4. The most predominant behaviors for G3 were Stand Alert (summer $=56 \%$; winter $=24 \%$ ) and Locomote (summer $=6 \%$; winter $=2 \%$ ), and for G4, the most predominant behaviors were also Stand Alert (summer $=31 \%$; winter $=9 \%)$ and Locomote $($ summer $=11 \%$; winter $=6 \%)$. No significant differences were found for behavioral diversity and the combined behavioral states of Inactive and Abnormal. In addition, there were no significant differences for Play or Abnormal behavior for individuals and the group when comparing summer to winter (Table 3).

\section{Recumbency Data}

No significant differences were found in the recumbency data between individuals $(\mathrm{G} 1, p=$ $0.198 ; \mathrm{G} 2, p=0.586 ; \mathrm{G} 3, p=0.595 ; \mathrm{G} 4, p=0.409$ ) or for the group when comparing summer and winter (Table 2). However, there was a significant positive correlation for time spent recumbent per hour for each 24-hour day measured in the summer and winter, $r=0.51, p=0.010$ (Figure 3). 


\section{FGM Analysis Data}

No significant differences were found for individual FGM concentrations between the seasons $(\mathrm{G} 1, p=0.636 ; \mathrm{G} 2, p=0.513 ; \mathrm{G} 3, p=0.679 ; \mathrm{G} 4, p=0.539)$ (Table 2). Figure 4 displays the average FGM concentrations $(\mathrm{ng} / \mathrm{g})$ for each giraffe for both seasons. Average FGM concentrations for the group were $2306.67 \mathrm{ng} / \mathrm{g}(S D=1194.51 \mathrm{ng} / \mathrm{g})$ in the summer and $4261.64 \mathrm{ng} / \mathrm{g}(S D=1332.04 \mathrm{ng} / \mathrm{g}) \mathrm{in}$ the winter (Figure 4).

\section{Discussion}

The purpose of this study was to examine seasonal differences in giraffe welfare exhibited at the Chicago Zoological Society - Brookfield Zoo. Although there were no significant results for the group between summer and winter, there was individual variation observed between the giraffe when comparing their behavior during the different seasons. One giraffe engaged in more Active Forage behavior in the winter than in the summer, and two giraffe engaged in more Active Non-Forage behavior in the summer than in the winter. No other significant differences were found, suggesting that, overall, there were no major impacts on animal welfare. We understand that interpretation of the data is limited due to having only one month to represent an entire season, as well as examining only one season each for summer and winter. However, allowing a comprehensive look at welfare with a small amount of individuals revealed significant results, thus, using these combined methods for future studies can help gain more information on seasonal welfare of giraffe.

Activity level was expected to be higher in the outdoor exhibit utilized in the summer and two giraffe, a 25-year old female and a 2-year old male (G3 and G4), had higher rates of both locomotive and standing alert behaviors during that time. Similarly, locomotion was seen at higher rates in the outdoor exhibit for sifakas (Macedonia, 1987) and elephants (Posta et al., 2013). Although giraffe in wild populations do not have extensive locomotive patterns, they move from one food source to another and a larger yard during the summer may encourage the giraffe to walk to different areas of the outdoor exhibit (Bashaw et al., 2001; Posta et al., 2013). The higher rate of standing alert behavior could also be due to the distinct difference of stimuli in the outdoor exhibit versus the indoor exhibit. The outdoor enclosure most likely provided more diverse stimuli, which would require the giraffe to be more alert and dynamic to their surroundings as a result (Bashaw et al., 2001; Hoff, Forthman, \& Maple, 1994).

A 10-year old female (G2) displayed a higher rate of Active Forage in the winter compared to summer. Similarly, Posta et al. (2013) saw two elephants spend more time feeding in their indoor exhibit during the winter. The authors suggested that this behavior was due to the subjects being provided higher quality diets in a smaller environment; therefore, the elephants would not have to search for their food but stay engaged in feeding activities (Posta et al., 2013). Similarly, the giraffe in the current study were provided with more enrichment devices with grain feed in their indoor enclosure during the winter, as well as being offered non-leafy branches (as opposed to leafy branches in the summer) in which they spent more time feeding on and stripping bark. Although enclosure size could be a possible factor to explain the increase in foraging activity in the indoor exhibit for our one giraffe, further research should continue to examine if changes in diet based on season affects foraging behavior in giraffe. With the small sample size used in this study, we cannot assume that the results from the individuals in this study represent all giraffe in professionally managed care; however, this information may be useful for zoo management to understand foraging behavior of giraffe between varying seasons.

Although recumbency rates were not significantly different between individuals or for the entire group, giraffe recumbency during the daytime was highest during the winter. Similarly, Veasey, Waran, and Young (1996) examined activity budgets of 19 giraffe in four zoos and found the only significant difference in behavior across the four environments was the duration of lying down (i.e., recumbency). Veasey et al. (1996) suggested that the difference most likely occurred because of the varying substrate 
between the four zoos, in which lying down was seen most with one individual in a grassy environment and no lying down was seen in hard-surfaced (paved) environments. This relationship was seen in cattle, in which the subjects spent significantly less time lying down on concrete substrate (Haley, de Passillé, \& Rushen, 2001). Although the different types of substrate between the indoor and outdoor exhibit could have led to this result, recumbency rates in this study were not significant but should be considered for future studies. However, there was a significant correlation between time spent recumbent per hour in each 24-hour day measured in the summer and winter, which suggests that the individuals in this study were recumbent in similar patterns for both seasons. In a similar study to Veasey et al. (1996), Bashaw (2011) saw consistent behavioral activity of four females between two different institutions and between indoor and outdoor exhibits. The author argued that environmental variables will alter an animal's behavior only if the changes will defer the animal's ability to meet its physical and mental needs; therefore, activity patterns (including recumbency levels) could essentially be consistent across all environments with the same criteria of care and management (Bashaw, 2011). Furthermore, if the exhibits were providing a similar standard of care for the giraffe in the current study, then we should have seen not only recumbency levels, but all behavioral activity, being consistent across both seasons. Because this was not the case due to individual variation, further information including recumbency data is needed to hopefully aid an understanding of how differences in housing can impact overall welfare.

The individual behavioral variation seen in this study demonstrates that welfare can vary within a species even when the individuals in the group are experiencing the same environment (Hill \& Broom, 2009; Hosey, Melfi, \& Pankhurst, 2013). Individuals of the same species in zoos may have different individual histories that could impact their ability to adapt to varying environments (Hill \& Broom, 2009). These differences can be referred to as "personality" or behavioral temperament in individuals, if observed consistently over time and context (Freeman \& Gosling, 2010; Gosling, 2001). Personality studies are becoming more common in zoo research as more institutions are utilizing individual behavioral differences as a tool towards understanding animal welfare and management (Powell \& Gartner, 2011; Watters \& Powell, 2012; Wielebnowski, 1999). Although there are species-specific characteristics that aid with an animal's response to the environment, the results of the current study showing individual variation demonstrate the importance of evaluating welfare at an individual level to meet each individual's behavioral needs.

The current study aimed to examine differences in giraffe welfare based on season. Although there were some significant differences observed, overall, there appeared to be little impact on giraffe welfare when comparing welfare measures from summer to winter. However, individual variation seen in this study should not be overlooked, as current trends in animal welfare research are moving towards the direction of assessing overall welfare of individual animals instead of species as a whole (Whitham \& Wielebnowski, 2013). Information gained from this study can aid with animal care and management. Two out of the four individuals displayed higher levels of locomotion in the summer compared to winter, therefore training less active animals to walk from one location to another in the winter or increased enrichment opportunities at different locations within the exhibit may alleviate this difference. Further research is needed with a larger sample size, as well as examining other factors such as age, sex, and social factors to better understand the impacts of seasonal exhibits on individual giraffe welfare. In addition, we understand that seasons can vary over time, therefore using more months to represent summer and winter and repeating with multiple seasons should be addressed in future studies. Ideally future studies will also include multiple observers such that reliability of behavioral observations can be established. We hope this study can help guide future research and serve as a model for other species with similar conditions.

\section{Acknowledgements}

The authors would like to thank Bill Zeigler for initiating this project and his continued support. We thank the Women's Board of the Chicago Zoological Society for their financial support. We would also like to thank Joan Wiczek for her time and assistance with the making of the giraffe bands. Finally, 
we would like to thank Amy Roberts, Joan Daniels, and entire giraffe animal care staff at the Chicago Zoological Society - Brookfield Zoo for making this study possible.

\section{References}

Altmann, J. (1974). Observational study of behavior: Sampling methods. Behaviour, 49, 227-266.

Baird, B. A., Kuhar, C. W., Lukas, K. E., Amendolagine, L. A., Fuller, G. A.,... Schook, M. W. (2016).

Program animal welfare: Using behavioral and physiological measures to assess the well-being of animals used for education programs in zoos. Applied Animal Behaviour Science, 176, 150-162.

Bashaw, M. J. (2011). Consistency of captive giraffe behavior under two different management regimes. Zoo Biology, 30, 371-378.

Bashaw, M. J., Sicks, F., Palme, R., Schwarzenberger, F., Tordiffe, A. S., \& Ganswindt, A. (2016). Non-invasive assessment of adrenocortical activity as a measure of stress in giraffe (Giraffa camelopardalis). BMC Veterinary Research, 12, 235.

Bashaw, M. J., Tarou, L. R., Maki, T. S., \& Maple, T. L. (2001). A survey assessment of variables related to stereotypy in captive giraffe and okapi. Applied Animal Behaviour Science, 73, 235-247.

Bergeron, R., Badnell-Waters, A. J., Lambton, S., \& Mason, G. (2006). Stereotypic oral behaviour in captive ungulates: Foraging, diet and gastrointestinal function. In: G. Mason \& J. Rushen (Eds.), Stereotypic animal behaviour: Fundamentals and applications to welfare (pp. 19-41). Wallingford: CAB International.

Chinnadurai, S. K., Millspaugh, J. J., Matthews, W. S., Canter, K., Slotow, R., ...Woods, R. J. (2009). Validation of fecal glucocorticoid metabolite assays for South African herbivores. The Journal of Wildife Management, 73, 1014-1020.

Dugard, P., File, P., \& Todman, J. (2012). Single-case and small-n experimental designs: A practical guide to randomization tests (2nd ed.). New York, NY: Routledge Academics.

Duggan, G., Burn, C. C., \& Clauss, M. (2015). Nocturnal behavior in captive giraffe (Giraffa camelopardalis) — a pilot study. Zoo Biology, 35, 14-18.

Fanson, K., \& Wielebnowski, N. (2013). Effect of housing and husbandry practices on adrenocortical activity in captive Canada lynx (Lynx canadensis). Animal Welfare, 22, 159-165.

Freeman, H. D., \& Gosling, S. D. (2010). Personality in nonhuman primates: A review and evaluation of past research. American Journal of Primatology, 72, 653-671.

Gibbons, J., Medrano-Galarza, C., de Passillé, A. M., \& Rushen, J. (2012). Lying laterality and the effect of IceTag data loggers on lying behaviour of dairy cows. Applied Animal Behaviour Science, 136, 104-107.

Gosling, S. D. (2001). From mice to men: What can we learn about personality from animal research? Psychological Bulletin, 127, 45-86.

Hacker, C. (2015). The examination of enrichment using space and food for African elephants (Loxodonta africana) at the San Diego Zoo Safari Park. Retrieved from Western Kentucky University TopSCHOLAR (Paper 1547).

Haley, D. B., de Passillé, A. M., \& Rushen, J. (2001). Assessing cow comfort: Effects of two floor types and two tie stall designs on the behaviour of lactating dairy cows. Applied Animal Behaviour Science, 71, 105-117.

Haley, D. B., Rushen, J., \& de Passillé, A. M. (2000). Behavioural indicators of cow comfort: Activity and resting behaviour of dairy cows in two types of housing. Canadian Journal of Animal Science, 80, 257-263.

Hassall, S. A., Ward, W. R., \& Murray, R. D. (1993). Effects of lameness on the behaviour of cows during the summer. The Veterinary Record, 132, 578-580.

Hatt, J. M., Schaub, D., Wanner, M., Wettstein, H. R., Flach, E. J., ... Clauss, M. (2005). Energy and fibre intake in a group of captive giraffe (Giraffa camelopardalis) offered increasing amounts of browse. Journal of Veterinary Medicine Series A, 52, 485-490.

Hill, S. P., \& Broom, D. M. (2009). Measuring zoo animal welfare: Theory and practice. Zoo Biology, 28, 531-544.

Hoff, M. P., Forthman, D. L., \& Maple, T. L. (1994). Dyadic interactions of infant lowland gorillas in an outdoor exhibit compared to an indoor holding area. Zoo Biology, 13, 245-256.

Hoff, M. P., Powell, D. M., Lukas, K. E., \& Maple, T. L. (1997). Individual and social behavior of lowland gorillas in outdoor exhibits compared with indoor holding areas. Applied Animal Behaviour Science, 54, 359-370.

Holdgate, M. R., Meehan, C. L., Hogan, J. N., Miller, L. J., Rushen, J.,... Shepherdson, D. J. (2016). Recumbence behavior in zoo elephants: Determination of patterns and frequency of recumbent rest and associated environmental and social factors. PloS ONE, 11, 1-19.

Hosey, G., Melfi, V., \& Pankhurst, S. (2013). Zoo animals: Behaviour, management, and welfare. Oxford, UK: 
Oxford University Press.

Hummel, J., Clauss, M., Baxter, E., Flach, E. J., \& Johansen, K. (2006). The influence of roughage intake on the occurrence of oral disturbances in captive giraffids. Zoo Animal Nutrition, 3, 235-252.

Kratochwill, T. R. (Ed.). (2013). Single subject research: Strategies for evaluating change. New York, NY: Academic Press.

Ladewig, J., \& Smidt, D. (1989). Behavior, episodic secretion of cortisol, and adrenocortical reactivity in bulls subjected to tethering. Hormones and Behavior, 23, 344-360.

Lukas, K. E., Hoff, M. P., \& Maple, T. L. (2003). Gorilla behavior in response to systematic alternation between zoo enclosures. Applied Animal Behaviour Science, 81, 367-386.

Macedonia, J. M. (1987). Effects of housing differences upon activity budgets in captive sifakas (Propithecus verreauxi). Zoo Biology, 6, 55-67.

Mallapur, A., Qureshi, Q., \& Chellam, R. (2002). Enclosure design and space utilization by Indian leopards (Panthera pardus) in four zoos in southern India. Journal of Applied Animal Welfare Science, 5, 111-124.

Medrano-Galarza, C., Gibbons, J., Wagner, S., de Passillé, A. M., \& Rushen, J. (2012). Behavioral changes in dairy cows with mastitis. Journal of Dairy Science, 95, 6994-7002.

Mellen, J., \& Sevenich MacPhee, M. (2001). Philosophy of environmental enrichment: Past, present, and future. Zoo Biology, 20, 211-226.

Miller, L. J., Pisacane, C. B., \& Vicino, G. A. (2016). Relationship between behavioural diversity and faecal glucocorticoid metabolites: A case study with cheetahs (Acinonyx jubatus). Animal Welfare, 25, 325-329.

Millspaugh, J. J., \& Washburn, B. E. (2004). Use of fecal glucocorticoid metabolite measures in conservation biology research: Considerations for application and interpretation. General and Comparative Endocrinology, 138, 189-199.

Millspaugh, J. J., Woods, R. J., Hunt, K. E., Raedeke, K. J., Brundige, G. C., ...Wasser, S. K. (2001). Fecal glucocorticoid assays and the physiological stress response in elk. Wildlife Society Bulletin, 899-907.

Morrow, C. J., Kolver, E. S., Verkerk, G. A., \& Matthews, L. R. (2002). Fecal glucocorticoid metabolites as a measure of adrenal activity in dairy cattle. General and Comparative Endocrinology, 126, 229-241.

Myers, G. (2004). The giraffe in captivity: Diet and nutrition. In A. Burgess (Ed.), The giraffe husbandry resource manual (pp. 116-126). Silver Springs, MD: Association of Zoos and Aquariums Antelope/Giraffe Taxon Advisory Group.

Novak, M.A., O’Neill, P., \& Suomi, S. J. (1992). Adjustments and adaptations to indoor and outdoor environments: Continuity and change in young adult rhesus monkeys. American Journal of Primatology, 28, 125-138.

Orban, D. A., Siegford, J. M., \& Snider, R. J. (2016). Effects of guest feeding programs on captive giraffe behavior. Zoo Biology, 35, 157-166.

Owen, M. A., Czekala, N. M., Swaisgood, R. R., Steinman, K., \& Lindburg, D. G. (2005). Seasonal and diurnal dynamics of glucocorticoids and behavior in giant pandas. Ursus, 16, 208-221.

Pellew, R. A. (1984). The feeding ecology of a selective browser, the giraffe (Giraffa camelopardalis tippelskirchi). Journal of Zoology, 202, 57-81.

Posta, B., Huber, R., \& Moore III, D. E. (2013). The effects of housing on zoo elephant behavior: A quantitative case study of diurnal and seasonal variation. International Journal of Comparative Psychology, 26, 37-52.

Powell, D. M., \& Gartner, M. C. (2011). Applications of personality to the management and conservation of nonhuman animals. In M. Inoue Murayama, S. Kawamura, \& A. Weiss (Eds.), Primatology monographs: From genes to animal behavior (pp. 185-199). Tokyo, Japan: Springer.

Ross, S. R., Calcutt, S., Schapiro, S. J., \& Hau, J. (2011). Space use selectivity by chimpanzees and gorillas in an indoor-outdoor enclosure. American Journal of Primatology, 73, 197-208.

Ross, S. R., Schapiro, S. J., Hau, J., \& Lukas, K. E. (2009). Space use as an indicator of enclosure appropriateness: A novel measure of captive animal welfare. Applied Animal Behaviour Science, 121, 42-50.

Ross, S. R., \& Shender, M. A. (2016). Daily travel distances of zoo-housed chimpanzees and gorillas: Implications for welfare assessments and space requirements. Primates, 57, 395-401.

Sapolsky, R. M. (1992). Neuroendocrinology of the stress response. Behavioral Endocrinology, 1, $287-324$.

Schaub, D., Clauss, M., Flach, E. J., Wettstein, H. R., Tack, C., \& Hatt, J. M. (2004). Influence of physical and chemical composition of diet on oral stereotypies in captive giraffes (Giraffa camelopardalis). Proceedings from The European Association of Zoo and Wildlife Veterinarians. 
Schwarzenberger, F. (2007). The many uses of non-invasive faecal steroid monitoring in zoo and wildlife species. International Zoo Yearbook, 41, 52-74.

Shannon, C. E., \& Weaver, W. (1949). The mathematical theory of communication. Urbana, IL: University of Illinois Press.

Shepherdson, D. J., Carlstead, K. C., \& Wielebnowski, N. (2004). Cross-institutional assessment of stress responses in zoo animals using longitudinal monitoring of faecal corticoids and behaviour. Animal Welfare, 13, S105-S114.

Shepherdson, D., Lewis, K. D., Carlstead, K., Bauman, J., \& Perrin, N. (2013). Individual and environmental factors associated with stereotypic behavior and fecal glucocorticoid metabolite levels in zoo housed polar bears. Applied Animal Behaviour Science, 147, 268-277.

Swaisgood, R. R. (2007). Current status and future directions of applied behavioral research for animal welfare and conservation. Applied Animal Behaviour Science, 102, 139-162.

Trunkfield, H. R., \& Broom, D. M. (1990). The welfare of calves during handling and transport. Applied Animal Behaviour Science, 28, 135-152.

Tucker, C. B., Weary, D. M., \& Fraser, D. (2004). Free-stall dimensions: Effects on preference and stall usage. Journal of Dairy Science, 87, 1208-1216.

Veasey, J. S., Waran, N. K., \& Young, R. J. (1996). On comparing the behaviour of zoo housed animals with wild conspecifics as a welfare indicator, using the giraffe (Giraffa camelopardalis) as a model. Animal Welfare, $5,139-153$.

Walker, M., Diez-Leon, M., \& Mason, G. (2014). Animal welfare science: Recent publication trends and future research priorities. International Journal of Comparative Psychology, 27, 80-100.

Watters, J. V., \& Powell, D. M. (2012). Measuring animal personality for use in population management in zoos: Suggested methods and rationale. Zoo Biology, 31, 1-12.

Whitham, J. C., \& Wielebnowski, N. (2013). New directions for zoo animal welfare science. Applied Animal Behaviour Science, 147, 247-260.

Wielebnowski, N. C. (1999). Behavioral differences as predictors of breeding status in captive cheetahs. Zoo Biology, 18, 335-349.

Wielebnowski, N. (2003). Stress and distress: Evaluating their impact for the well-being of zoo animals. Journal of the American Veterinary Medical Association, 223, 973-977.

Wielebnowski, N., \& Brown, J. L. (2003). Proceedings from The Society for Behavioral Neuroendocrinology '03: Applying noninvasive hormone monitoring and behavioral studies to address management issues in threatened and endangered felids. Cincinnati, OH: University of Cincinnati.

Yadav, R., Mohan, K., Kumar, V., Sarkar, M., Nitu, K., ...Prakash, B. S. (2013). Development and validation of a sensitive enzyme immunoassay (EIA) for blood plasma cortisol in female cattle, buffaloes, and goats. Domestic Animal Endocrinology, 45, 72-78. 